IJMMS 31:11 (2002) 639-650

PII. S0161171202203142

http://ijmms.hindawi.com

(c) Hindawi Publishing Corp.

\title{
CONVEX DYNAMICS IN HELE-SHAW CELLS
}

\author{
DMITRI PROKHOROV and ALEXANDER VASIL'EV
}

\author{
Received 15 March 2002
}

\begin{abstract}
We study geometric properties of a contracting bubble driven by a homogeneous source at infinity and surface tension. The properties that are preserved during the time evolution are under consideration. In particular, we study convex dynamics of the bubble and prove that the rate of the area change is controlled by variation of the bubble logarithmic capacity. Next we consider injection through a single finite source and study some isoperimetric inequalities that correspond to the convex and $\alpha$-convex dynamics.
\end{abstract}

2000 Mathematics Subject Classification: 30C45, 76S05, 76D99, 35Q35, 30C35.

1. Hele-Shaw problem. We are concerned with the one-phase Hele-Shaw problem in two space dimensions. Hele-Shaw [13] was the first who described in 1898 the motion of a fluid in a narrow gap between two parallel plates. A significant contribution after his work was made in 1945 by Polubarinova-Kochina [26, 27] and Galin [11], and then, by Saffman and Taylor [31] who discovered viscous fingering in 1958. New interest to this problem is reflected, for example, in a more than 600 item bibliography made by Gillow and Howison in the workshop of Hele-Shaw free boundary problems (http://www.maths.ox.ac.uk/ howison/Hele-Shaw).

In our first case the phase domain of a moving viscous fluid is the complement to a simply connected bounded domain occupied by an inviscid fluid (or an ideal gas). We call it the outer problem and the problem of suction/injection into a bounded phase domain is called the inner problem. Two driving mechanisms are considered. The principal one is suction/injection through a single well at infinity. Another one is surface tension. When surface tension is zero, the main feature of the process is cusp formation at the moving interface at a finite blow-up time. Examples of such a scenario have been known since 1945 [11, 26, 27] and a classification of cusps has been proposed in [14]. Now we present a simple version of the one-phase planar Hele-Shaw moving boundary problem with suction/injection through a single well at infinity. We denote by $\Omega(t)$ a simply connected domain in the phase $z$-plane occupied by the fluid at instant $t$ that contains $\infty$ as an interior point. The complement to $\Omega(t)$ is a simply connected bounded domain $D(t)$. We assume the sink/source to be of strength $Q(t)$ that in general depends on time. We will never use this dependence throughout our paper nevertheless we mention that $Q(t)$ can be reduced to a constant $Q$ by a suitable change of variables. The dimensionless pressure $p$ is scaled so that 0 corresponds to the atmospheric pressure. The dimensionless model of a moving viscous incompressible fluid is described by the potential flow with a velocity field $\mathbf{V}=\left(V_{1}, V_{2}\right)$. The pressure $p$ gives rise to the fluid velocity $\mathbf{V}=-K \nabla p$, where $K=$ $h^{2} / 12 \mu$ is a positive constant, $h$ is the cell gap, and $\mu$ is the viscosity of the fluid 
(see, e.g., [25]). We set $K$ by a suitable scaling to be equal to 1 and put $\Gamma(t) \equiv \partial \Omega(t)$. With $z=x+i y$ a parameterization of $\Gamma(t)$ is given by the equation $\phi(x, y, t) \equiv \phi(z, t)=0$. The initial situation is represented at the instant $t=0$ as $\Omega(0)=\Omega_{0}$, and the boundary $\partial \Omega_{0}=\Gamma(0) \equiv \Gamma_{0}$ is defined parametrically by an implicit function $\phi(x, y, 0)=0$. Since we consider incompressible fluid, we have the equality $\nabla \cdot V=0$, which implies that $p$ is a harmonic function

$$
\nabla^{2} p=0, \quad z=x+i y \in \Omega(t) \backslash\{\infty\} .
$$

The zero-surface-tension dynamical boundary condition is given by

$$
p(z, t)=0 \quad \text { as } z \in \Gamma(t) .
$$

The resulting motion of the free boundary $\Gamma(t)$ is given by the velocity field $\mathbf{V}$ on $\Gamma(t)$ with the normal velocity in the outward direction

$$
v_{n}=\left.\mathbf{V}\right|_{\Gamma(t)} \cdot \hat{n}(t)
$$

where $\hat{n}(t)$ is the unit outer normal vector to $\Gamma(t)$. This condition means that the boundary is formed by the same set of particles at any time. Near infinity we have $p \sim Q \log \sqrt{x^{2}+y^{2}}$ as $x, y \rightarrow \infty$ that relates to the homogeneous flow. The value of $Q$ corresponds to the rate of bubble release caused by air extraction, $Q<0$ in the case of a contructing bubble and $Q>0$ otherwise.

We can regard this model as the dynamics of an extending/contracting bubble in a Hele-Shaw cell. This model has various applications in the boundary value problems of gas mechanics, problems of metal or polymer swamping, and so forth, where the air viscosity is neglected. More about this problem is found in [6, 17, 22].

One of the typical properties of problem (1.1) is the fact that its character depends on the direction of evolution of the free boundary. In the case of fluid suction $(Q>0)$ the problem is ill-posed in the Hadamard sense. This means that an arbitrary small perturbation of the boundary $\Gamma_{0}$ of the initial domain $\Omega_{0}$ can produce an $O(1)$-order deformation of $\Gamma(t)$ in an arbitrary small time $t$. The injection problem $(Q<0)$ is well-posed at least for the weak solution (Elliott and Janowsky [5]).

One of the main features of problem (1.1) is as follows: starting with an analytic boundary $\Gamma_{0}$ we obtain a one-parameter $(t)$ chain of the solutions $p(z, t)$ (and equivalently $\phi(x, y, t))$ that exist during a period $t \in\left[0, t_{0}\right)$ developing possible cusps at the boundary $\Gamma(t)$ in a blow-up time $t_{0}$. It is known [36] that in the Hele-Shaw problem (1.1) the classical solution exists locally in time. Recently [12, 16, 30], it became clear that this model could be interpreted as a particular case of the abstract Cauchy problem, thus, the classical solvability (locally in time) may be proved using the nonlinear abstract Cauchy-Kovalevskaya theorem.

In most practical experiments the zero-surface-tension process is never observed. An approximation of the practical situation is given by introducing surface tension. At the same time the nonzero-surface-tension model regularizes the ill-posed problem. That is why the consideration of the surface tension influence is of importance.

The model with nonzero surface tension is reduced to the condition for the pressure $p$ on the boundary given by the product of the curvature $\varkappa$ of the boundary and surface 
tension $\gamma>0$. We rewrite problem (1.1) with the following new conditions:

$$
\begin{array}{cc}
\nabla^{2} p=0, & \text { in } z \in \Omega(t), \\
p=\gamma \varkappa(z), & \text { on } z \in \Gamma(t), \\
v_{n}=-\frac{\partial p}{\partial n}, & \text { on } z \in \Gamma(t) .
\end{array}
$$

A similar problem appears in metallurgy in the description of the motion of phase boundaries by capillarity and diffusion [22]. Condition (1.3) is found in [31] (it is also known as the Laplace-Young boundary condition, see, e.g., [2], or the GibbsThomson condition [16]). It takes into account how surface tension modifies the pressure through the boundary interface.

The problem of the solution existence in the nonzero-surface-tension case is more difficult. Duchon and Robert [3] proved the local existence in time of the weak solution for all $\gamma$. Recently, Prokert [29] obtained even global existence in time and exponential decay of the solution near equilibrium for bounded domains. The results are obtained in Sobolev spaces $W^{2, s}$ with sufficiently big $s$. We refer the reader to the works by Escher and Simonett [7, 8, 9, 10] who proved the local existence, uniqueness, and regularity of classical solutions to one- and two-phase Hele-Shaw problems with surface tension when the initial domain has a smooth boundary. The global existence in the case of the phase domain close to a disk was proved in [8]. More about results on existence for general parabolic problems can be found in [10].

Mathematical treatment for the case of the zero-surface-tension model of a contracting bubble was presented in [6]. In particular, the problem of the limiting configuration was solved. It was proved that the moving boundary tends to a finite number of points that gave the minimum to a certain potential. There an interesting problem was proposed: to describe domains whose dynamics presents only one limiting point. Howison [17] proved that a contracting elliptic bubble has the homothetic dynamics to a point (in particular, this is obvious for a circular one). Entov and Ètingof [6] have shown that a contracting bubble which is convex at the initial instant preserves this property until the moment when its boundary reduces to a point. This type of domains is called simple in [6].

We will generalize this result proving that if the initial bubble is starlike with respect to a fixed inner point, then locally in time this property is preserved. Moreover, we will give some estimates of the rate of the change of the boundary capacity by the rate of the area change.

2. Polubarinova-Galin equation with surface tension. In order to present the equation for the moving boundary $\Gamma(t)$, we introduce the auxiliar parametric domain which is the exterior part of the unit disk, and by the Riemann mapping theorem there exists a unique conformal univalent map $F(\zeta, t)$ from the domain $U^{*}=\{\zeta:|\zeta|>1\}$ onto the phase domain $\Omega(t)$, such that $F(\zeta, t)=a \zeta+a_{0}+a_{-1} / \zeta+\cdots, a>0$. The function $F(\zeta, 0)=F_{0}(\zeta)$ produces a parameterization of $\Gamma_{0}=\left\{F_{0}\left(e^{i \theta}\right), \theta \in[0,2 \pi)\right\}$ and the moving boundary is parameterized by $\Gamma(t)=\left\{F\left(e^{i \theta}, t\right), \theta \in[0,2 \pi)\right\}$. The normal velocity $v_{n}$ of $\Gamma(t)$ in the outward (with respect to $\Omega(t)$ ) direction is given 
by $v_{n}=-\partial p / \partial n$. Later on, throughout the paper we use the notations $\dot{F}=\partial F / \partial t$, $F^{\prime}=\partial F / \partial \zeta$.

We introduce the complex potential $W(z, t), z \in \Omega(t)$, so that $\operatorname{Re} W(z, t)=p(z, t)$. Then $\nabla p=\bar{W}^{\prime}$, and near infinity we have the expansion

$$
W(z, t)=\frac{Q}{2 \pi} \log z+w_{0}(z, t), \quad \text { as } z \sim \infty,
$$

where $w_{0}(z, t)$ is a regular function in $\Omega(t)$.

The normal outer vector at the boundary $\Gamma(t)$ is given by the formula

$$
\hat{n}=-\zeta \frac{F^{\prime}}{\left|F^{\prime}\right|}, \quad \zeta \in \partial U^{*}
$$

Therefore, the normal velocity is obtained as

$$
v_{n}=\mathbf{V} \cdot \hat{n}=-\operatorname{Re}\left(\frac{\partial W}{\partial z} \zeta \frac{F^{\prime}}{\left|F^{\prime}\right|}\right)
$$

The superposition $W \circ F(\zeta, t)$ is an analytic function in $U^{*} \backslash\{\infty\}$ and has a logarithmic singularity about infinity. Its real part solves the Dirichlet problems (1.2) and (1.3), therefore,

$$
W \circ F(\zeta, t)=\frac{Q}{2 \pi} \log \zeta-\frac{\gamma}{2 \pi} \int_{0}^{2 \pi} x\left(e^{i \theta}, t\right) \frac{e^{i \theta}+\zeta}{e^{i \theta}-\zeta} d \theta+i C
$$

Differentiating (2.4) we get

$$
\zeta \frac{\partial W}{\partial z} f^{\prime}(\zeta, t)=\frac{Q}{2 \pi}-\frac{\gamma}{\pi} \int_{0}^{2 \pi} \frac{\varkappa\left(e^{i \theta}\right) \zeta e^{i \theta}}{\left(e^{i \theta}-\zeta\right)^{2}} d \theta, \quad \zeta \in U^{*} .
$$

Integrating by parts we obtain

$$
\zeta \frac{\partial W}{\partial z} f^{\prime}(\zeta, t)=\frac{Q}{2 \pi}-\frac{\gamma}{2 \pi i} \int_{0}^{2 \pi} \frac{\partial \varkappa}{\partial \theta} \frac{e^{i \theta}+\zeta}{e^{i \theta}-\zeta} d \theta .
$$

On the other hand, we have $v_{n}=-\operatorname{Re} \dot{\bar{F}} \overline{e^{i \theta} F^{\prime} /\left|F^{\prime}\right|}$, and applying the Sokhotskiı̌-Plemelj formulae [23] we, finally, get

$$
\operatorname{Re} \dot{F}(\zeta, t) \overline{\zeta F^{\prime}(\zeta, t)}=\frac{Q}{2 \pi}-\gamma\left(H\left[i \frac{\partial \varkappa}{\partial \theta}\right](\theta)\right),
$$

$\zeta=e^{i \theta}$, where the Hilbert transform in (2.7) is of the form

$$
H[\psi](\theta) \equiv-\frac{1}{\pi} \mathbf{p} \cdot \mathbf{v} \cdot \theta \int_{0}^{2 \pi} \frac{\psi\left(e^{i \theta^{\prime}}\right) d \theta^{\prime}}{1-e^{i\left(\theta-\theta^{\prime}\right)}} .
$$

Galin [11] and Polubarinova-Kochina [26, 27] first have derived (2.7) for $\gamma=0$ and gave rise to deep investigation in this direction. So, (2.7) for $\gamma=0$ is known as the Polubarinova-Galin equation (see, e.g., [14, 19, 20]). 
Equation (2.7) yields a Löwner-Kufarev type equation making use of the SchwarzPoisson formula

$$
\dot{F}=\zeta F^{\prime} p_{F}(\zeta, t), \quad \zeta \in U^{*},
$$

where

$$
p_{F}(\zeta, t)=\frac{1}{2 \pi} \int_{0}^{2 \pi} \frac{1}{\left|f^{\prime}\left(e^{i \theta}, t\right)\right|^{2}}\left(\frac{Q}{2 \pi}-\gamma H\left[i \frac{\partial \varkappa}{\partial \theta}\right](\theta)\right) \frac{e^{i \theta}+\zeta}{e^{i \theta}-\zeta} d \theta .
$$

We call (2.9) a Löwner-Kufarev type equation because of the analogy with the linear partial differential equation that describes the homotopy deformation of a simply connected univalent domain to the initial one (see, e.g., [1, 4, 28]). The classical LöwnerKufarev equation produces a subordination Löwner chain. Unlike the classical LöwnerKufarev equation, equation (2.9) is not quasilinear, contains an integral operator $p_{F}$, and produces a special type of chain (nonsubordinate in general). Nevertheless, it is quickly noticed that in the case of extending bubble $(Q>0)$ and small surface tension $\gamma$ we have $\operatorname{Re} \dot{F}(\zeta, t) \overline{\zeta F^{\prime}(\zeta, t)}>0$, and the Löwner-Kufarev theory implies that the chain of the domains $\Omega(t)$ is subordinate, that is, $\Omega(s) \subset \Omega(t)$ for $s>t$.

Of course, (2.7) tends to the equation for the zero-surface-tension model as $\gamma \rightarrow 0$. But it turns out that the solution in the limiting $\gamma$-surface-tension case need not always be the corresponding zero-surface-tension solution (see the discussion in [32, 33, 34]). This means that starting with a domain $\Omega(0) \equiv \Omega(0, \gamma)$ we come to the domain $\Omega(t, \gamma)$ at an instant $t$ using surface tension $\gamma$ and to the domain $\Omega(t)$ at the same instant $t$ in the zero-surface-tension model. Then the domain $\lim _{\gamma \rightarrow 0} \Omega(t, \gamma)=\Omega(t, 0)$ is not necessarily the same as $\Omega(t)$ (see numerical evidence in [2, 24]). Obviously, the nonzero-surface-tension model never produces cusps, moreover, starting with an analytic boundary $\Gamma_{0}$ the curves $\Gamma(t)$ remain analytic during the time of existence of the solution.

3. Convex and starlike contracting bubble. A simply connected domain $\Omega$ on the Riemann sphere, $\infty \in \Omega, 0 \notin \Omega$ is said to be starlike if each ray starting at the origin intersects $\Omega$ in a ray. Of course, the complement of $\Omega$ to the Riemann sphere is starlike with respect to the origin. Let a univalent function $F$ map the exterior part $U^{*}$ of the unit disk onto $\Omega$, so that $F(\zeta) \neq 0$ for any $\zeta \in U^{*}$ and $F(\zeta)=a \zeta+a_{0}+\sum_{n=1}^{\infty} a_{n} \zeta^{-n}$ about infinity. If $\Omega$ is starlike, then the function $F$ is also called starlike, $F \in \Sigma^{*}$. A necessary and sufficient condition for a locally univalent function $F(\zeta), \zeta \in U^{*}$, with the above normalization to be univalent and starlike is the following inequality:

$$
\operatorname{Re} \frac{\zeta F^{\prime}(\zeta)}{F(\zeta)}>0, \quad \zeta \in U^{*}
$$

We denote by $t_{0}$ the blow-up time and we consider the dynamics of the contracting bubble in the Hele-Shaw cell. Let $F(\zeta, t)$ be the family of functions satisfying the Polubarinova-Galin equation (2.7) and $D_{0}=\hat{\mathbb{C}} \backslash \Omega_{0}, D(t)=\hat{\mathbb{C}} \backslash \Omega(t)$.

THEOREM 3.1. Let $Q<0$ and surface tension $\gamma$ be sufficiently small. If the initial domain $\Omega_{0}$ (and $D_{0}$ ) is starlike with the analytic boundary, then there exists $t=t(\gamma) \leq$ $t_{0}$, such that the family of domains $\Omega(t)$ (in sequel, the family of univalent functions 
$F(\zeta, t)$ and the domains $D(t))$ preserves this property during the time $t \in[0, t(\gamma)]$. In particular, for $\gamma=0$ the family $\Omega(t)$ preserves this property in so far as the solution exists and $0 \in D(t)$.

Proof. We have that the contracting bubble contains the origin and is starlike with respect to the origin at the initial instant. If a starlike function $F(\zeta)=a \zeta+$ $a_{0}+a_{-1} / \zeta+\cdots \in \Sigma^{*}$ is defined outside of the unit disk, then the function $f(\zeta)=$ $1 / F(1 / \zeta)$ is holomorphic in the unit disk $U$ and starlike $\left(f \in S^{*}\right)$ with respect to the origin. The inequality

$$
\operatorname{Re} \frac{\zeta f^{\prime}(\zeta)}{f(\zeta)}>0, \quad \zeta \in U
$$

provides the necessary and sufficient condition for the function $f$ to be univalent and starlike.

Equation (2.7) can be rewritten in terms of this holomorphic function as

$$
\operatorname{Re} \dot{f}(\zeta, t) \overline{\zeta f^{\prime}(\zeta, t)}=-|f(\zeta, t)|^{4}\left(\frac{Q}{2 \pi}-\gamma\left(H\left[i \frac{\partial \varkappa}{\partial \theta}\right](\theta)\right)\right),
$$

$|\zeta|=1, Q<0$. If we consider $f$ in the closure of $U$, then the inequality sign in (3.2) can be replaced by $(\geq)$ where equality can be attained for $|\zeta|=1$.

We suppose that there exists a critical map $f \in S^{*}$, which means that the image of $U$ under the map $\zeta f^{\prime}(\zeta, t) / f(\zeta, t),|\zeta| \geq 1$ touches the imaginary axis, say there exist such $t^{\prime} \geq 0$ and $\zeta_{0}=e^{i \theta_{0}}$, that

$$
\arg \frac{\zeta_{0} f^{\prime}\left(\zeta_{0}, t^{\prime}\right)}{f\left(\zeta_{0}, t^{\prime}\right)}=\frac{\pi}{2} \quad\left(\text { or }-\frac{\pi}{2}\right)
$$

and for any $\varepsilon>0$ there are such $t>t^{\prime}$ and $\theta \in\left(\theta_{0}-\varepsilon, \theta_{0}+\varepsilon\right)$ that

$$
\arg \frac{e^{i \theta} f^{\prime}\left(e^{i \theta}, t\right)}{f\left(e^{i \theta}, t\right)} \geq \frac{\pi}{2} \quad\left(\text { or } \leq-\frac{\pi}{2}\right)
$$

For definiteness we put the sign (+) in (3.4). Without loss of generality, assume $t^{\prime}=0$. Since $f^{\prime}\left(e^{i \theta}, t\right) \neq 0$, our assumption about the sign in (3.4) yields

$$
\operatorname{Im} \frac{\zeta_{0} f^{\prime}\left(\zeta_{0}, 0\right)}{f\left(\zeta_{0}, 0\right)}>0
$$

(the negative case is considered similarly).

Since $\zeta_{0}$ is a critical point and the image of $U$ under the mapping $\zeta f^{\prime}(\zeta, 0) / f(\zeta, 0)$ touches the positive imaginary axis at the point $\zeta_{0}=e^{i \theta_{0}}$, we deduce that

$$
\begin{array}{r}
\left.\frac{\partial}{\partial \theta} \arg \frac{e^{i \theta} f^{\prime}\left(e^{i \theta}, 0\right)}{f\left(e^{i \theta}, 0\right)}\right|_{\theta=\theta_{0}}=0, \\
\left.\frac{\partial}{\partial r} \arg \frac{r e^{i \theta_{0}} f^{\prime}\left(r e^{i \theta_{0}}, 0\right)}{f\left(r e^{i \theta}, 0\right)}\right|_{r=1^{-}} \geq 0 .
\end{array}
$$


Calculation gives

$$
\begin{aligned}
& \operatorname{Re}\left[1+\frac{\zeta_{0} f^{\prime \prime}\left(\zeta_{0}, 0\right)}{f^{\prime}\left(\zeta_{0}, 0\right)}-\frac{\zeta_{0} f^{\prime}\left(\zeta_{0}, 0\right)}{f\left(\zeta_{0}, 0\right)}\right]=0, \\
& \operatorname{Im}\left[1+\frac{\zeta_{0} f^{\prime \prime}\left(\zeta_{0}, 0\right)}{f^{\prime}\left(\zeta_{0}, 0\right)}-\frac{\zeta_{0} f^{\prime}\left(\zeta_{0}, 0\right)}{f\left(\zeta_{0}, 0\right)}\right] \geq 0 .
\end{aligned}
$$

We derive

$$
\frac{\partial}{\partial t} \arg \frac{\zeta f^{\prime}(\zeta, t)}{f(\zeta, t)}=\operatorname{Im} \frac{\partial}{\partial t} \log \frac{f^{\prime}(\zeta, t)}{f(\zeta, t)}=\operatorname{Im}\left(\frac{(\partial / \partial t) f^{\prime}(\zeta, t)}{f^{\prime}(\zeta, t)}-\frac{(\partial / \partial t) f(\zeta, t)}{f(\zeta, t)}\right) .
$$

We now differentiate (3.3) with respect to $\theta$. Since the left-hand side is real analytic with respect to $\theta$ and the solution to (2.7), and therefore to (3.3), exists and is unique, the right-hand side is differentiable and its derivative is bounded on $[0,2 \pi]$. Denote by

$$
A(\theta, t):=\frac{\partial}{\partial \theta} H\left[i \frac{\partial \varkappa}{\partial \theta}\right](\theta)
$$

Then we have

$$
\begin{aligned}
\operatorname{Im}\left(\overline{F^{\prime}(\zeta, t)} \frac{\partial}{\partial t} F^{\prime}(\zeta, t)-\overline{\zeta F^{\prime}(\zeta, t)} \dot{F}(\zeta, t)-\overline{\zeta^{2} F^{\prime \prime}(\zeta, t)} \dot{F}(\zeta, t)\right) \\
\quad=-4|f|^{4} \operatorname{Im} \frac{\zeta f^{\prime}}{f}\left(\frac{Q}{2 \pi}-\gamma H\left[i \frac{\partial \varkappa}{\partial \theta}\right](\theta)\right)+\gamma|f|^{4} A(\theta, t),
\end{aligned}
$$

for $\zeta=e^{i \theta}$. This equality is equivalent to the following:

$$
\begin{aligned}
\left|f^{\prime}(\zeta, t)\right|^{2} & \operatorname{Im}\left(\frac{(\partial / \partial t) f^{\prime}(\zeta, t)}{f^{\prime}(\zeta, t)}-\frac{(\partial / \partial t) f(\zeta, t)}{f(\zeta, t)}\right) \\
= & \operatorname{Im} \overline{\zeta f^{\prime}(\zeta, t)} \dot{f}(\zeta, t)\left(\frac{\overline{\zeta f^{\prime \prime}(\zeta, t)}}{f^{\prime}(\zeta, t)}-\frac{\zeta f^{\prime}(\zeta, t)}{f(\zeta, t)}+1\right) \\
& +4|f|^{4} \operatorname{Im} \frac{\zeta f^{\prime}}{f}\left(\frac{Q}{2 \pi}-\gamma H\left[i \frac{\partial \varkappa}{\partial \theta}\right](\theta)\right)-\gamma|f|^{4} A(\theta, t)
\end{aligned}
$$

Substituting (3.3), (3.8), and (3.10) in the latter expression we finally have

$$
\begin{aligned}
\frac{\partial}{\partial t} \arg & \left.\frac{\zeta f^{\prime}(\zeta, t)}{f(\zeta, t)}\right|_{\zeta=e^{i \theta_{0}, t=0}} \\
= & \frac{Q\left|f\left(e^{i \theta_{0}}, 0\right)\right|^{4}}{\left|f^{\prime}\left(e^{i \theta_{0}}, 0\right)\right|^{2}} \operatorname{Im}\left(1-\frac{e^{i \theta_{0}} f^{\prime}\left(e^{i \theta_{0}}, 0\right)}{f\left(e^{i \theta_{0}}, 0\right)}+\frac{e^{i \theta_{0}} f^{\prime \prime}\left(e^{i \theta_{0}}, 0\right)}{f^{\prime}\left(e^{i \theta_{0}}, 0\right)}+6 \frac{e^{i \theta_{0}} f^{\prime}\left(e^{i \theta_{0}}, 0\right)}{f\left(e^{i \theta_{0}}, 0\right)}\right) \\
& -\gamma \frac{\left|f\left(e^{i \theta_{0}}, 0\right)\right|^{4}}{\left|f^{\prime}\left(e^{i \theta_{0}}, 0\right)\right|^{2}}\left(4 \operatorname{Im} \frac{\zeta f^{\prime}}{f} H\left[i \frac{\partial \mathcal{\varkappa}}{\partial \theta}\right](\theta)+A(\theta, t)\right) .
\end{aligned}
$$


The right-hand side of this equality is strictly negative for small $\gamma$ because of (3.6) and (3.9). Therefore,

$$
\arg \frac{e^{i \theta} f^{\prime}\left(e^{i \theta}, t\right)}{f\left(e^{i \theta}, t\right)}<\frac{\pi}{2}
$$

for $t>0$ (close to 0 ) in some neighbourhood of $\theta_{0}$. This contradicts the assumption of the existence of the critical map and, equivalently, the hypothesis that $\Omega(t)$ fails to be starlike for some $t>0$ and ends the proof.

Of course, we can shift any inner point $z_{0}$ of the bubble to the origin by a linear transform. So the above result can be rewritten as follows: if we find a point $z_{0}$ in the initial bubble $D_{0}$ with respect to which $D_{0}$ is starlike, then the domains $D(t)$ are also starlike with respect to the same point $z_{0}$ during the existence of the solution or up to the time when $z_{0} \in \Gamma(t)$. This means that if $D_{0}$ is simple, $z_{0}$ is a limiting point at which $D(t)$ contracts, and $D_{0}$ is starlike with respect to $z_{0}$, then $D(t)$ remains starlike up to the instant when all air is removed (there exist nonconvex simple domains, see [6]).

In particular, a convex domain $D_{0}$ is starlike with respect to any point from $D_{0}$, and therefore, the convex dynamics is also preserved that was proved earlier in [6].

Now, we present an isoperimetric inequality which implies that the rate of the area variation of a contracting bubble of zero surface tension is controlled by the rate of the variation of its capacity.

Proposition 3.2. Denote by $S(t)$ the area of a contracting bubble $D(t)$, and $\gamma=0$. Then $\dot{S} \geq 2 \pi a \dot{a}$, where $a=\operatorname{cap} D(t)$.

Proof. A simple application of the Green theorem implies that the rate of the area change is expressed as $\dot{S}=Q$. Let $Q<0$. From (2.7) we deduce that

$$
\dot{a}=a \frac{1}{4 \pi^{2}} \int_{0}^{2 \pi} \frac{Q}{\left|F^{\prime}\left(e^{i \theta}, t\right)\right|^{2}} d \theta \leq a \frac{1}{4 \pi^{2}} \int_{0}^{2 \pi} \operatorname{Re} \frac{Q}{F^{\prime}\left(e^{i \theta}, t\right)^{2}} d \theta=\frac{Q}{2 \pi a}=\frac{\dot{S}}{2 \pi a},
$$

where $a=F^{\prime}(\infty, t)$.

4. Convex dynamics and integral means. Now we discuss the problem of injection of a fluid within the complex plane through a finite source that can be thought of as the origin. The governing equations are of the form

$$
\begin{gathered}
\nabla^{2} p=-Q \delta(0), \quad \text { in } z \in \Omega(t), \\
p=\gamma \varkappa(z), \quad \text { on } z \in \Gamma(t), \\
v_{n}=-\frac{\partial p}{\partial n}, \quad \text { on } z \in \Gamma(t) .
\end{gathered}
$$

Here $p$ stands for pressure in the phase simply connected bounded domain $\Omega(t)$ occupied by the fluid, $\boldsymbol{x}$ is the mean curvature, and $\gamma$ is the surface tension. $Q$ is negative and corresponds to the strengthen of the source. The problem of injection is well-posed, $Q<0$. We refer the reader to [14, 18, 20, 21, 24] where a lot of curious features concerning the problem of suction can be found. 
We consider the auxiliar Riemann map $z=f(\zeta, t)$ from the unit disk $U$ onto the phase domain $\Omega(t), f(0, t) \equiv 0, f^{\prime}(0, t)>0$. The Polubarinova-Galin equation for the moving boundary $\Gamma(t)=\partial \Omega(t)$ is given as

$$
\operatorname{Re} \dot{f}(\zeta, t) \overline{\zeta f^{\prime}(\zeta, t)}=-\frac{Q}{2 \pi}+\gamma\left(H\left[i \frac{\partial \varkappa}{\partial \theta}\right](\theta)\right),
$$

$\zeta=e^{i \theta}$, where the Hilbert transform in (4.2) is of the form

$$
H[\psi](\theta) \equiv \frac{1}{\pi} \mathbf{p} \cdot \mathbf{v} \cdot \theta \int_{0}^{2 \pi} \frac{\psi\left(e^{i \theta^{\prime}}\right) d \theta^{\prime}}{1-e^{i\left(\theta-\theta^{\prime}\right)}} .
$$

In [15] we prove that if the initial domain $\Omega_{0}$ is starlike with respect to the origin, then during the whole time of the existence of the solution to (4.2) the domains $\Omega(t)$ remain to be starlike for $\gamma=0$, or locally in time for $\gamma$ sufficiently small [35]. Of course, if the initial domain is convex, then in general, the convex dynamics is not preserved even in the next instant. But locally in time we can guarantee the convex dynamics if the initial domain is $\alpha$-convex. The necessary and sufficient condition for the domain $\Omega$ to be convex is the inequality for the Riemann map

$$
\operatorname{Re}\left(1+\frac{\zeta f^{\prime \prime}(\zeta)}{f^{\prime}(\zeta)}\right)>0, \quad \zeta \in U
$$

A domain (or equivalently a function) is said to be $\alpha$-convex if the zero in the above inequality is replaced by a positive number $\alpha \in(0,1]$.

Proposition 4.1. Denote by $S(t)$ the area of the phase domain $\Omega(t)$. Then $\dot{S}=-Q$.

This obvious proposition follows from the statement of the problem as well as from Green's theorem.

Proposition 4.2. Let a univalent map $z=f(\zeta)$ be $\alpha$-convex in $U$ and let $f$ have the angular derivatives almost everywhere in the unit circle. Then,

$$
\frac{1}{2 \pi} \int_{0}^{2 \pi} \frac{1}{\left|f^{\prime}\left(e^{i \theta}\right)\right|^{2}} d \theta \leq \frac{2^{8(1-\alpha)}}{\pi} \mathbf{B}\left(\frac{5}{2}-2 \alpha, \frac{5}{2}-2 \alpha\right),
$$

where $\mathbf{B}(\cdot, \cdot)$ stands for the Euler beta-function. The inequality is sharp. In particular,

$$
\frac{1}{2 \pi} \int_{0}^{2 \pi} \frac{1}{\left|f^{\prime}\left(e^{i \theta}\right)\right|^{2}} d \theta \leq \frac{4^{1-4 \alpha}}{2 \pi} \frac{(3-4 \alpha)(1-4 \alpha)}{(1-\alpha)(1-2 \alpha)} \mathbf{B}\left(\frac{1}{2}-2 \alpha, \frac{1}{2}-2 \alpha\right)
$$

for $0 \leq \alpha<1 / 4$.

PROoF. If a function $f$ is $\alpha$-convex in $U$, then the analytic function $g(z) \equiv z f^{\prime}(z)$ is $\alpha$-starlike ( $S_{\alpha}^{*}$ ), that is, it satisfies inequality (3.2) replacing 0 by $\alpha$ in its right-hand side. Functions from $S_{\alpha}^{*}$ admit the following known integral representation:

$$
g(z) \in S_{\alpha}^{*} \Longleftrightarrow g(z)=z \exp \left\{-2(1-\alpha) \int_{-\pi}^{\pi} \log \left(1-e^{i \theta} z\right) d \mu(\theta)\right\},
$$

where $\mu(\theta)$ is a nondecreasing function of $\theta \in[-\pi, \pi]$ and $\int_{-\pi}^{\pi} d \mu(\theta)=1$. 
If $\mu(\theta)$ is a piecewise constant function, then we have a set of complex-valued functions $g_{n}(z)$ that admit the following representation:

$$
g_{n}(z)=\frac{z}{\prod_{k=1}^{n}\left(1-e^{i \theta_{k}} z\right)^{2(1-\alpha) \beta_{k}}} \in S_{\alpha}^{*}, \quad \theta_{k} \in[-\pi, \pi], \quad \beta_{k} \geq 0, \sum_{k=1}^{n} \beta_{k}=1 .
$$

Using the known properties of Stieltjes' integral and Vitali's theorem it is easy to show that the set of function (4.8) is dense in $S_{\alpha}^{*}$, that is, for every function $g(z) \in S_{\alpha}^{*}$ there exists a sequence $\left\{g_{n}(z)\right\}$ satisfying (4.8) that locally uniformly converges to $g(z)$ in $U$. Therefore, we need to prove our result for $g(z)=g_{n}(z)$.

Now, we present a chain of inequalities

$$
\begin{aligned}
\frac{1}{2 \pi} \int_{0}^{2 \pi} \frac{1}{\left|g_{n}\left(e^{i \theta}\right)\right|^{2}} d \theta & =\frac{1}{2 \pi} \int_{0}^{2 \pi} \prod_{k=1}^{n}\left|1-e^{i\left(\theta-\theta_{k}\right)}\right|^{4(1-\alpha) \beta_{k}} d \theta \\
& \leq \frac{1}{2 \pi} \int_{0}^{2 \pi} \sum_{k=1}^{n} \beta_{k}\left|1-e^{i\left(\theta-\theta_{k}\right)}\right|^{4(1-\alpha)} d \theta \\
& =\frac{1}{2 \pi} \sum_{k=1}^{n} \beta_{k} \int_{0}^{2 \pi}\left|1-e^{i\left(\theta-\theta_{k}\right)}\right|^{4(1-\alpha)} d \theta \\
& =\frac{1}{2 \pi} \int_{0}^{2 \pi}\left|1-e^{i \theta}\right|^{4(1-\alpha)} d \theta \\
& =\frac{4^{1-\alpha}}{2 \pi} \int_{0}^{2 \pi}(1-\cos \theta)^{2(1-\alpha)} d \theta \\
& =\frac{2^{8(1-\alpha)}}{\pi} \mathbf{B}\left(\frac{5}{2}-2 \alpha, \frac{5}{2}-2 \alpha\right) .
\end{aligned}
$$

The last assertion of Proposition 4.2 follows from the formulae of reduction of the beta-function.

The next theorem follows from Propositions 4.1 and 4.2 similarly to the proof of Proposition 3.2.

THEOREM 4.3. Let $\Omega(t)$ be a phase domain occupied by a fluid injected through the origin with surface tension $\gamma=0$, the area of $\Omega(t)$ be $S(t)$, and $a(t)$ be the conformal radius of $\Omega(t)$ with respect to the origin. Then $\dot{S} \leq 2 \pi a \dot{a}$. If, moreover, $\Omega(t)$ is $\alpha$-convex at an instant $t$, then

$$
\frac{2 \pi^{2} a \dot{a}}{2^{8(1-\alpha)} \mathbf{B}(5 / 2-2 \alpha, 5 / 2-2 \alpha)} \leq \dot{S} \leq 2 \pi a \dot{a} .
$$

ACKNOWLEDGMENTS. The first author is supported by the Russian Foundation for Basic Research, grant No. 01-01-00123 and the INTAS grant No. 99-00089; the second author is supported by FONDECYT, project No. 1010093; both authors are supported by FONDECYT, project No. 7010093.

\section{REFERENCES}

[1] I. A. Aleksandrov, Parametric Continuations in the Theory of Univalent Functions, Nauka, Moscow, 1976 (Russian). 
[2] H. D. Ceniceros, T. Y. Hou, and H. Si, Numerical study of Hele-Shaw flow with suction, Phys. Fluids 11 (1999), no. 9, 2471-2486.

[3] J. Duchon and R. Robert, Évolution d'une interface par capillarité et diffusion de volume. I. Existence locale en temps [Interface evolution by capillarity and volume diffusion. I. Local existence in time], Ann. Inst. H. Poincaré Anal. Non Linéaire 1 (1984), no. 5, 361-378 (French).

[4] P. L. Duren, Univalent Functions, Fundamental Principles of Mathematical Sciences, vol. 259, Springer-Verlag, New York, 1983.

[5] C. M. Elliott and V. Janovský, A variational inequality approach to Hele-Shaw flow with a moving boundary, Proc. Roy. Soc. Edinburgh Sect. A 88 (1981), no. 1-2, 93-107.

[6] V. M. Entov and P. I. Ètingof, Bubble contraction in Hele-Shaw cells, Quart. J. Mech. Appl. Math. 44 (1991), no. 4, 507-535.

[7] J. Escher and G. Simonett, On Hele-Shaw models with surface tension, Math. Res. Lett. 3 (1996), no. 4, 467-474.

[8] _ Classical solutions for Hele-Shaw models with surface tension, Adv. Differential Equations 2 (1997), no. 4, 619-642.

[9] _ A center manifold analysis for the Mullins-Sekerka model, J. Differential Equations 143 (1998), no. 2, 267-292.

[10]__ Moving surfaces and abstract parabolic evolution equations, Topics in Nonlinear Analysis, Progr. Nonlinear Differential Equations Appl., vol. 35, Birkhäuser, Basel, 1999, pp. 183-212.

[11] L. A. Galin, Unsteady filtration with a free surface, C. R. (Doklady) Acad. Sci. URSS (N.S.) 47 (1945), 246-249.

[12] B. Gustafsson, On a differential equation arising in a Hele-Shaw flow moving boundary problem, Ark. Mat. 22 (1984), no. 2, 251-268.

[13] H. S. Hele-Shaw, The flow of water, Nature 58 (1898), no. 1489, 33-36.

[14] Yu. E. Hohlov and S. D. Howison, On the classification of solutions to the zero-surfacetension model for Hele-Shaw free boundary flows, Quart. Appl. Math. 51 (1993), no. 4, 777-789.

[15] Yu. E. Hohlov, D. V. Prokhorov, and A. Yu. Vasil'ev, On geometrical properties of free boundaries in the Hele-Shaw flows moving boundary problem, Lobachevskii J. Math. 1 (1998), 3-12.

[16] Yu. E. Hohlov and M. Reissig, On classical solvability for the Hele-Shaw moving boundary problems with kinetic undercooling regularization, European J. Appl. Math. 6 (1995), no. 5, 421-439.

[17] S. D. Howison, Bubble growth in porous media and Hele-Shaw cells, Proc. Roy. Soc. Edinburgh Sect. A 102 (1986), no. 1-2, 141-148.

[18] _ C C C _ Cusp development in Hele-Shaw flow with a free surface, SIAM J. Appl. Math. 46 (1986), no. 1, 20-26.

[19] Complex variable methods in Hele-Shaw moving boundary problems, European J. Appl. Math. 3 (1992), no. 3, 209-224.

[20] S. D. Howison, A. A. Lacey, and J. R. Ockendon, Hele-Shaw free-boundary problems with suction, Quart. J. Mech. Appl. Math. 41 (1988), no. 2, 183-193.

[21] S. D. Howison, J. R. Ockendon, and A. A. Lacey, Singularity development in movingboundary problems, Quart. J. Mech. Appl. Math. 38 (1985), no. 3, 343-360.

[22] W. W. Mullins, Grain boundary grooving by volume diffusion, Trans. Metallurgical Society of AIME 218 (1960), 354-361.

[23] N. I. Muskhelishvili, Singular Integral Equations. Boundary Problems of Function Theory and Their Application to Mathematical Physics, P. Noordhoff N. V., Groningen, 1953.

[24] Q. Nie and F. R. Tian, Singularities in Hele-Shaw flows, SIAM J. Appl. Math. 58 (1998), no. 1, 34-54. 
[25] H. Ockendon and J. R. Ockendon, Viscous Flow, Cambridge Texts in Applied Mathematics, Cambridge University Press, Cambridge, 1995.

[26] P. Ya. Polubarinova-Kochina, Concerning unsteady motions in the theory of filtration, Prikl. Mat. Meh. 9 (1945), no. 1, 79-90 (Russian).

[27] On a problem of the motion of the contour of a petroleum shell, Dokl. Acad. Nauk USSR 47 (1945), no. 4, 254-257 (Russian).

[28] C. Pommerenke, Univalent Functions. With a Chapter on Quadratic Differentials by Gerd Jensen, Studia Mathematica/Mathematische Lehrbücher, vol. 25, Vandenhoeck \& Ruprecht, Göttingen, 1975.

[29] G. Prokert, Existence results for Hele-Shaw flow driven by surface tension, European J. Appl. Math. 9 (1998), no. 2, 195-221.

[30] M. Reissig and L. von Wolfersdorf, A simplified proof for a moving boundary problem for Hele-Shaw flows in the plane, Ark. Mat. 31 (1993), no. 1, 101-116.

[31] P. G. Saffman and G. Taylor, The penetration of a fluid into a porous medium or Hele-Shaw cell containing a more viscous liquid, Proc. Roy. Soc. London Ser. A 245 (1958), no. 281, 312-329.

[32] M. Siegel, S. Tanveer, and W.-S. Dai, Singular effects of surface tension in evolving HeleShaw flows, J. Fluid Mech. 323 (1996), 201-236.

[33] S. Tanveer, Evolution of Hele-Shaw interface for small surface tension, Philos. Trans. Roy. Soc. London Ser. A 343 (1993), no. 1668, 155-204.

[34] F.-R. Tian, Hele-Shaw problems in multidimensional spaces, J. Nonlinear Sci. 10 (2000), no. 2, 275-290.

[35] A. Vasil'ev and I. Markina, On the geometry of Hele-Shaw flows with small surface tension, Preprint MAT 2000/11, Universidad Técnica Federico Santa María, 2000.

[36] Yu. P. Vinogradov and P. P. Kufarev, On a problem of filtration, Akad. Nauk SSSR Prikl. Mat. Meh. 12 (1948), 181-198 (Russian).

DMitri Prokhorov: Department of Mathematics and Mechanics, Saratov State UniVERSITY, SARATOV 410026, RUSSIA

E-mail address: prokhorovdv@info.sgu.ru

AleXander Vasil'ev: Departamento de Matemática, UTFSM, CASIlla, 110-V ValParaíso, CHILE

E-mail address: a lexander.vasi1 i ev@mat.utfsm.c1 


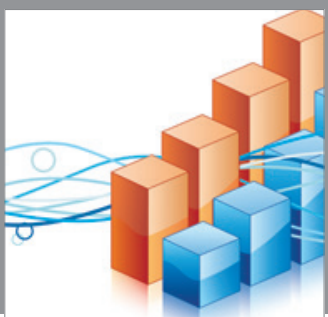

Advances in

Operations Research

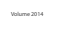

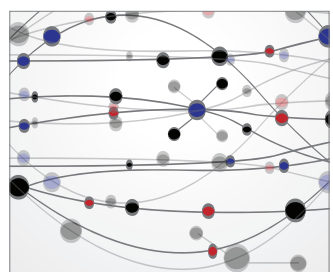

\section{The Scientific} World Journal
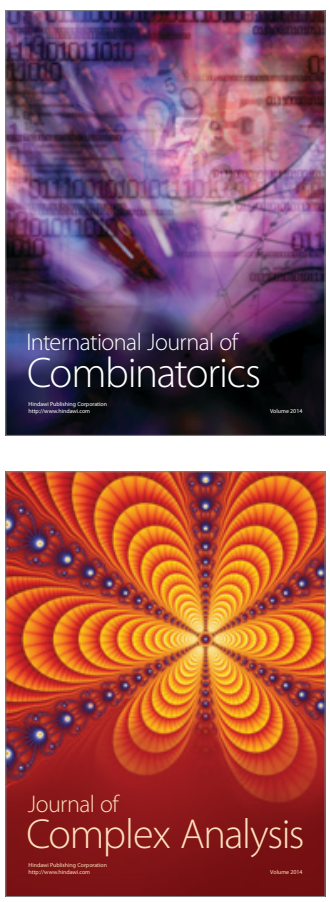

International Journal of

Mathematics and

Mathematical

Sciences
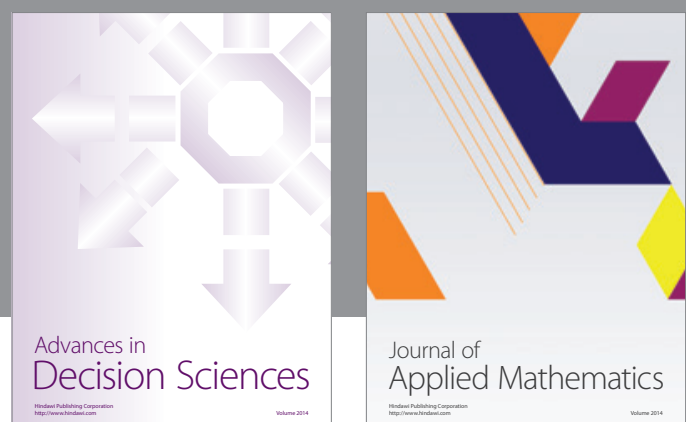

Journal of

Applied Mathematics
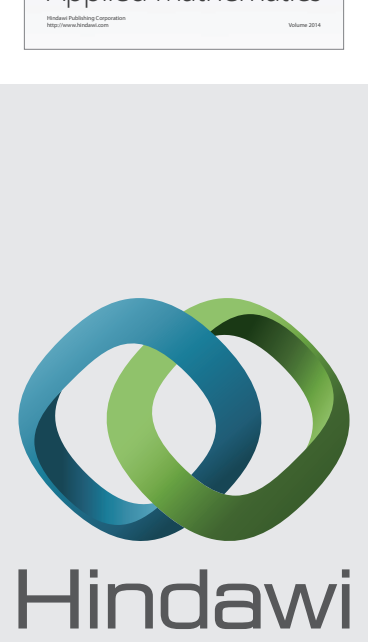

Submit your manuscripts at http://www.hindawi.com
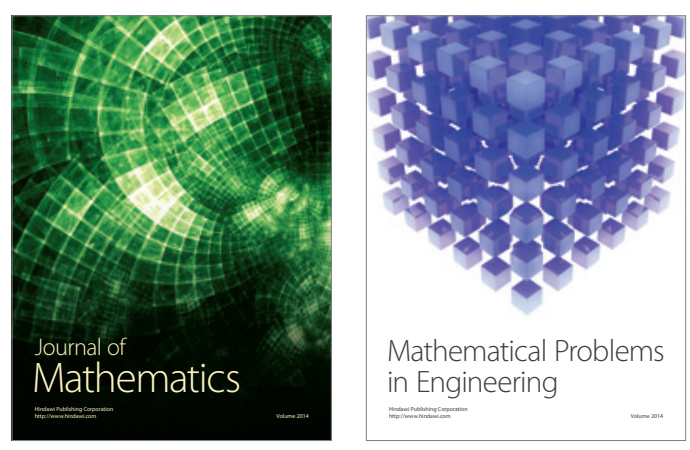

Mathematical Problems in Engineering
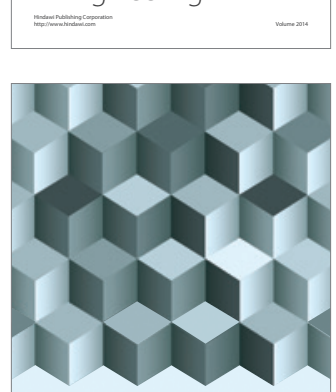

Journal of

Function Spaces
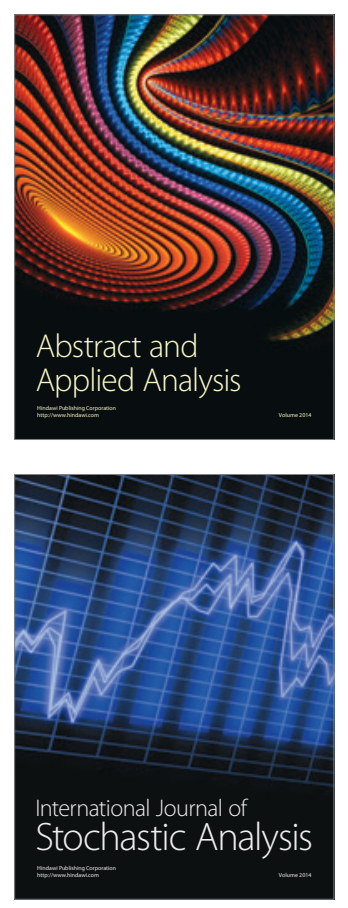

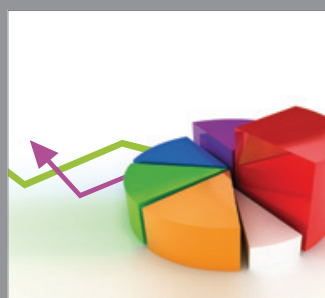

ournal of

Probability and Statistics

Promensencen
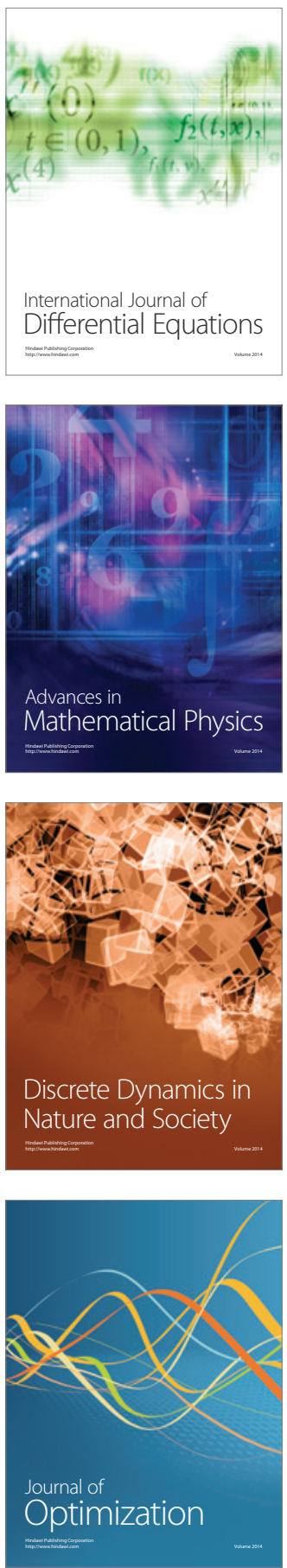